\title{
PROCEEDINGS OF THE FOURTH ANNUAL MEETING OF THE CENTRAL SOCIETY FOR CLINICAL RESEARCH HELD IN CHICAGO, NOVEMBER 20, 1931
}

The Influence of Work and of Thyroxine on the Metabolism of a Dog. By Walter M. Boothby, M.D. Rochester, Minn.

The effect of eleven intravenous injections of $10 \mathrm{mgm}$. of thyroxine each on the metabolism of a dog over a period of nine months was studied. The experiment-to be reported in full by Boothby, Buckley and Wilhelmj ( $\mathrm{J}$. Physiol.)-was divided into three periods. The first period, lasting eleven days, was without work and followed at least a year of confined cage life. In the second period of 115 days the dog was exercised on a treadmill. In the third period of sixty-four days the exercise was again omitted. Throughout the experiment the dog was on a constant weighed diet containing 2.93 grams nitrogen and 480 calories each day. The nitrogen in the urine averaged 2.65 grams and in the feces and hair shed into the cage 0.24 gram daily, totaling 2.89 grams daily. Within the limits of experimental error average nitrogen equilibrium was maintained in all three periods. Immediately following the injection of thyroxine there is a temporary negative nitrogen balance followed by a compensatory positive balance after which equilibrium is attained. During period (1) the average respiratory quotient for all days, except the first four after each injection of thyroxine, averaged 0.82 ; for period (2), 0.81 , and for period (3), 0.83 . The dog lost weight in period (2) on account of the exercise; as he was in nitrogen equilibrium the loss of weight must have been due to loss of fat; the heat value of the fat corresponding to the loss in weight was calculated to be equivalent to the energy of the extra work; it was also calculated that this amount of fat would lower the average respiratory quotient from 0.82 to 0.81 as found experimentally. Similarly, in period (3) the dog gained in weight due to deposition of fat as nitrogen equilibrium existed; the retention of this amount of fat would raise the average respiratory quotient from 0.82 to 0.83 , as found experimentally. During the first four days after injection of thyroxine the average respiratory quotient was decreased to an average of $0.77,0.75$, and 0.76 in the three periods, respectively; this decrease was greater than could be accounted for by the amount of fat needed to meet the increased energy requirements. The increased excretion of nitrogen immediately following administration of thyroxine was largely due to increased elimination of urea and to a slight extent to the elimination of creatine; the quantities of the other nitrogenous partition products did not vary significantly; the few days of compensating nitrogen retention was accounted for by a decrease in elimination of urea. The decay curve of thyroxine is more irregular in dogs than in human beings, but within the limits of experimental error it was consistent with the exponential form found in this laboratory for human beings. Following exercise on the treadmill the decay curve during period (2) decreased less rapidly than in the preliminary control period and this effect continued throughout the third period again without exercise. Under the conditions of this experiment the total calorigenic action of $10 \mathrm{mgm}$. thyroxine averaged 220 calories in period (1) without exercise, 449 calories in period (2) with exercise and 487 calories in period (3) without exercise. 
Experimental Exophthalmos and its Relation to the Pathogenesis of Exophthalmic Goiter. By HARRY B. FRIEDGOOD, M.D. (by invitation) and Allen M. Boyden (by invitation), Ann Arbor, Mich. (introduced by Cyrus C. Sturgis, M.D.).

Faradic stimulation of the right superior cervical sympathetic ganglion in three dogs resulted in unmistakable unilateral exophthalmos, dilatation of the pupil and slight lacrimation, but both palpebral fissures widened perceptibly, especially on the right. Resection of this ganglion induced rapid enophthalmos and myosis. These changes were definitely demonstrated by a series of motion pictures of the experiment.

Exophthalmos, lid-lag and "lid-spasm" are infrequently seen in conditions other than exophthalmic goiter, for example, in essential hypertension and the "functional" psychoneuroses. These eye signs are not diagnostic of Graves' syndrome, but represent objective phenomena indicative of the state of tonicity in the sympathetic nervous system.

Increased nervous tension, secondary to any cause whatsoever, may be responsible for a markedly elevated basal metabolic rate. The action of epinephrine, an activator of the sympathetic nervous system, is also characterized by a definite rise in the basal metabolic rate. These data suggest that the sympathetic nervous system may be at least partially responsible for the elevated oxygen consumption in exophthalmic goiter.

Tachycardia, tremor, abnormal sweating, emotional and vasomotor instability, exophthalmos and associated eye signs are among the cardinal signs of sympathetic nervous system hyperactivity. The predominance of these nervous disturbances and the intensity with which they develop in Graves' syndrome suggest that the sympathetic nervous system plays a paramount rôle in its pathogenesis.

Metabolic Studies During Morphine Withdrawal from a Human Addict. By Dwight C. Ensign (by invitation) and Frank J. Sladen, Detroit, Mich.

Clinical studies emphasize the psychic factor in explanation of the symptoms following withdrawal of morphine. Studies upon animals, in which typical addiction may be produced, relate these symptoms to certain metabolic changes especially affecting carbohydrates and water. Directly opposite results have been obtained by different workers. Studies upon human subjects have resulted in similarly varying results. The authors' opportunity for study included a prolonged period of observation of a patient with a minimal psychic factor. The plan of treatment, precluding knowledge of reduction, was successful in avoiding the "before-the-next-dose" abstinence reaction. We report findings (1) before withdrawal; (2) during reduction; and (3) during the period of major symptoms of abstinence. Correlation of symptoms of withdrawal and findings.

\section{A Clinical Study of the Cardiodynamics of Mitral Insufficiency and of Mitral}

Stenosis. By Harold FeIL, M.D., Cleveland, Ohio.

A brief discussion of the cardiodynamics in experimental acute mitral insufficiency and in acute mitral stenosis is presented. In a clinical study of fifteen cases of mitral insufficiency without signs of active infection or of failure the duration of the chief phases of systole and of total systole was normal. Twenty-one patients with clinical evidence of mitral stenosis were studied. Of twelve patients with normal mechanism eleven showed no deviation from the average duration of systole and of its chief phases; one patient with evidence of early cardiac failure had abbreviation of total systole and of ejection. Nine pa- 
tients with auricular fibrillation all showed shortening of the ejection phase and of total systole. Mitral insufficiency and mitral stenosis cause no change in the duration of systole and of its chief phases unless failure or auricular fibrillation are present when shortening of total systole and of ejection occur.

These findings are in agreement with findings in these experimental lesions and it may be assumed that the compensatory processes in animals probably operate identically in man.

Changes in the Electrocardiogram in the Course of Pericardial Effusion with Paracentesis and Pericardiotomy. By JoHn Harvey, M.D., and JoHN W. Scotr, M.D., Lexington, Ky.

In 1929 Scott, Feil and Katz reported the occurrence of the plateau type T wave characteristic of recent coronary occlusion in, (1) aneurysm ruptured into the pericardium and (2) purulent pericardial effusion. Since then considerable interest has been shown in the occurrence of such $\mathrm{T}$ waves in conditions other than coronary occlusion. Clinically it has been observed in pneumonia. In the experimental animal this type of $\mathrm{T}$ wave has been observed after coronary ligation, after injection of toxic material into the muscle of the ventricle, after a toxic dose of digitalis, after the injection of fluid into the pericardium and during induced general anoxemia.

Opportunity has been afforded to observe a case of pneumococcic pericarditis with massive effusion in which the plateau type $T$ wave was present. Daily electrocardiograms taken in the course of the illness extending over seventeen days, during which paracentesis and later pericardiotomy was done, show interesting changes with temporary return to normal five days before death. Autopsy showed neither coronary nor gross myocardial lesions.

It is thought that these observations may have some bearing upon the relative importance of increased intrapericardial pressure and anoxemia as the cause of such electrocardiographic changes.

Pneumopericardium in the Treatment of Pericarditis with Effusion. By M. H. Nathanson, M.D., Minneapolis, Minn.

The literature contains comparatively few references to the therapeutic use of air in the pericardial cavity, only about twenty cases having been published. In the present report two patients with the exudative type of tuberculous pericarditis were treated first by the removal of fluid and later by the removal of fluid and injection of air. One patient has been observed for fifteen months and at the present time has a low grade tuberculosis of the peritoneal cavity. The other patient was first treated in 1923 and at the present time is in apparent good health, although there is some enlargement of the heart.

A review of the literature and the experience in these two cases indicates the following:

1. Pneumopericardium can be carried out repeatedly without danger or discomfort to the patient.

2. The immediate result as evidenced by decrease in dyspnea and by increased diuresis is much better than that obtained by removal of the fluid alone.

3. As compared with simple removal of the fluid there is a distinct slowing in the return of the exudate with pneumopericardium.

4. The procedure permits a beautiful visualization on the roentgenogram of the pathology in the pericardium, the degree with which the sac is emptied by puncture, and the progress of the reaccumulation of the exudate.

5. Theoretically, the air, by keeping the inflamed surfaces apart, should reduce the mechanical irritation and also tend to prevent adhesions. This can only 
be true in a degree as several cases in the literature which came to autopsy showed extensive adhesive pericarditis and both patients in the present report show roentgenograms indicating adhesive pericarditis.

6. The possible curative effect of air on the exudate comparable to that which has been described in tuberculous peritonitis is suggested by the distinct improvement in the patient's general condition, the change of the fluid from hemorrhagic to clear, and the reduction in the number of tubercle bacilli in those cases in which they had been isolated.

\section{Liver Damage Incident to Diets Low in Protein. By M. Herbert Barker, M.D., Chicago, Ill.}

Dogs placed on a diet theoretically adequate in calories, minerals and vitamins but deficient in nitrogen gradually developed a fatty degenerative process in the liver. This fatty replacement became extensive if the diet was continued. A hypercholesterolemia developed early in the process and continued up until the animal's appetite began to fail. If protein was gradually added, the animal would usually recover. Repair of the liver has been extremely slow and functional tests and biopsies show extensive parenchymatous damage still present after six months.

Weight Loss and Nitrogen Excretion of Obese Patients on Low Calorie Diets with High and Low Quantities of Protein. By Robert W. Keeton, M.D., and Dorothy Dickson (by invitation), Chicago, Ill.

Obese patients weighing from 100 to $150 \mathrm{kgm}$. were hospitalized and studied in the following manner. During a preliminary period of ten to fourteen days they were placed on diets containing 90 grams of protein and calories equivalent to their basal requirements. This allowed the patient's metabolism to become adjusted to this level of protein and his weight to become roughly stationary. He was then placed for a period of six weeks on a diet containing calories 30 to 48 per cent below his basal level and 90 grams of protein. This was followed by a second period of six weeks in which the diet contained 14 grams of protein and basal minus 30 per cent calories; and a third period similar to the first.

Eight patients were studied according to this plan. Data has been secured on twelve other patients who were unable to remain through such a long experimental period.

Conclusions: 1. Patients living on diets containing 90 grams of protein and calories from 30 to 50 per cent below their basal requirements for six weeks, ten weeks and twelve weeks continued to lose weight uniformly throughout these periods.

2. Patients lost weight as well on diets containing 14 grams of protein, calories remaining the same as on diets with 90 grams of protein.

3. Weight loss occurred when the patients were in positive and negative balance. The fluctuations in weight loss were not correlated with fluctuations in nitrogen balance.

4. These patients requisition extra calories from the stored fat and so maintain their nitrogen equilibrium in the face of a severe caloric deficit.

5. The patients were more comfortable on the high protein diets.

Study of Disorders of Duodenal Motility. By R. L. SEnSEnich, M.D., South Bend, Ind.

Mechanical interference with normal duodenal motility, due to organic causes, produces retention and characteristic symptoms, and suggests that other phe- 
nomena might result from temporary functional disturbances of motor activity. Roentgenologists have described various phenomena, said to be due to duodenal irritability, but without noting association of definite clinical symptomatology. Physiologists have in turn reported symptoms elicited by experimental irritation or stimulation of the undiseased duodenal wall. The study here reported was for the purpose of further investigating the association of common types of digestive discomfort with definite functional disorders of duodenal motility.

Patients have been observed over a period of years and possible organic causes have been eliminated. Association of certain symptoms with certain definite duodenal motor phenomena have been noted. These observations have demonstrated a tendency on the part of each patient to reproduce physical signs, subjective symptoms, and x-ray findings, according to his individual pattern, whenever subjected to sufficient nervous stress or fatigue.

Characteristic clinical symptoms observed, varied from mild sensation of digestive malfunction to nausea and vomiting or violent cephalalgia. Disturbances of duodenal motility varied in degree and in the portion of the duodenum involved. Hypermotility, changing points of irritability with delay, or spastic distal portion with duodenal distention and gastric retention were observed.

The diagnosis must be made by fluoroscopic observation continued throughout a sufficient period of time, when symptoms are present. This may be during transit of the barium meal or two to four hours later, when a small amount of barium must again be given to demonstrate the duodenal motility if the previous meal has passed. The significant diagnostic evidence is that the symptoms complained of are associated with the motor phenomena observed and are relieved when they cease. The use of small amounts of barium, suspended in water, best demonstrates the variations of duodenal motility. The recent work of Berg in bringing the surface of the mucosa into relief in roentgen studies gives promise of being of some help in differentiating organic duodenal conditions.

Tumors of the Suprarenal Gland. By RALPH G. BALL, M.D. (by invitation) and Leonard G. Rowntree, M.D., Rochester, Minn.

The various types of suprarenal tumors seen in the Mayo Clinic are discussed and correlated with the cases in the literature. The series includes cases of pseudohermaphroditism, hirsutism and virilism. The clinical manifestations clear up subsequent to operation in many instances. On the other hand, death from suprarenal failure has resulted in numerous cases following operation. This it may be possible to avoid in the future with the use of the new suprarenal cortical hormone.

Comparative study of the cases of paragangliomata of Mayo-Rowntree, Shipley-Pincoff, and Porter and Porter reveals striking analogy in the history and clinical findings, in the operative results, and in the pathology of the tumors. Cases of continuous hypertension associated with medullary and cortical tumors with and without the changes in body configuration are reviewed. Twenty-nine cases have been collected from the literature in which there seems to be a relationship between suprarenal tumor and hypertension.

In relation to hypertension we must consider, first, that at least one active principle has been isolated from the suprarenal gland which has a tremendous effect on blood pressure; second, that with tumors of this gland hypertension is extremely common and may be either intermittent or continuous. In at least one unoperated case, hypertension of the intermittent type has become continuous. Removal of the tumor has resulted in relief from hypertension in several instances. The lack of evidence of pathologic change in the suprarenal glands in 
hypertension is no more striking than the lack of evidence of pathologic change in the pancreas in cases of diabetes mellitus. We are forced, then, to the view that there is a small and special group of cases of suprarenal hypertension, or that the suprarenals participate directly or indirectly in the pathogenesis of hypertension.

An Analysis of the Lymphocyte in Pulmonary Tuberculosis. By B. K. WISEman, M.D., and C. A. DoAn, M.D., Columbus, Ohio.

The characteristics of the blood-cells in pulmonary tuberculosis have recently assumed increased importance. Medlar, among others, has called attention to the significance of variations in the neutrophilic leucocytes and Sabin and Doan have emphasized the importance of the monocyte, its qualitative changes and the value of the numerical relationship existing between both monocytes and lymphocytes as expressed by the $M / L$ index. Work done in our laboratory has indicated that certain qualitative changes occurring in the lymphocyte constitute criteria of a life cycle in that cell in many ways comparable to similar maturative phenomena in the other blood cells. This has led to the recognition of a lymphocyte formula that is rather constant in health. Utilizing these criteria, it has been possible to analyze the qualitative changes in the lymphocyte portion of the $\mathrm{M} / \mathrm{L}$ ratio. Such studies in both clinical and animal tuberculosis indicate that the lymphopenia usually occurring in cases progressing unfavorably is due to an inhibition of the maturative forces in the lymphocytogenic tissues, and is reflected in the blood by an abnormal lymphocyte formula. Thus it has been found that while the $\mathrm{M} / \mathrm{L}$ ratio is a valuable expression of an important numerical relationship between monocytes and lymphocytes, more complete information as to the progress of the tuberculous lesion can be adduced from an analysis of the qualitative changes in the lymphocytes and monocytes separately.

The Problem of Tuberculous Reinfection. By R. G. BLoch, M.D., and B. F. FRANCIS, M.D. (by invitation), Chicago, Ill.

The extensive use of the roentgen ray in the study of patients in our Chest Clinic has produced the material presented here. Our findings support the recently reported evidence of others for the theory that the infra-clavicular region of the lung is the most common location of the earliest lesion of active and progressive tuberculosis. This lesion is found on the $\mathrm{x}$-ray plate before physical signs are manifest, often while symptoms are still very mild or even absent. The early lesion is more often found in the axillary half than in the medial half of the lung field, and varies from a few millimeters to several centimeters in extent. It also varies from the definitely circumscribed and homogeneous lesion to a diffuse one mottled in appearance. Occasionally excavation occurs and then it appears as an isolated cavity. The prognosis of this type of lesion varies. In many patients serial $x$-ray plates show a progressive disappearance even though no treatment is instituted, in others a long period of bed rest or collapse therapy is necessary. Of course, only a comparatively small proportion of patients are first seen in this early stage; most of them show more advanced disease on the roentgenogram. In these instances, when the apices are involved, the problem of the location of the early lesion arises. This question is discussed. Tuberculosis confined to an apex does exist and the pathological relationship between this type and the infraclavicular infiltration is also discussed.

In many of our patients who had had recent contact with tuberculosis, the early infiltration was found on the $\mathbf{x}$-ray even though no symptoms or physical 
signs of any kind were present. This led to the most important consideration of the study, the significance of the lesion from the public health standpoint, and the part played by exogenous reinfection in the etiology of the adult type of pulmonary tuberculosis. In our opinion it indicates first, that great attention should be paid to the isolation of patients with open tuberculosis from contact with adults, especially young adults, and second, that a good roentgenogram should be made of the chests of all contacts regardless of the presence or absence of symptoms or signs.

The Copper, Non-Hemoglobinous Iron and Antitrypsin Contents of the Blood Serum in Disease. By Arthur Locke, Ph.D., E. R. MaIN, M.S., D. O. Rosbash, B.S. (by invitation) and Edwin F. Hirsch, M.D., Chicago, Ill.

The iron content of the hemoglobin-free serum of normal persons from whom food has been withheld for 12 hours varies from an average value of 0.9 microgram per cc. for young men to a value of 0.8 microgram for young women. Values of 1.4-1.5 microgram and above are found in the cord blood of newly born infants. Variable values are observed in disease. Conditions associated with fever usually are characterized by a lowered serum iron level, and conditions associated with a retention of bilirubin with an increased iron level. The high serum iron level associated with pernicious anemia becomes corrected following the administration of liver extract.

The copper content of the blood serum of young men appears to be lower and more variable than that of young women, ranging from a maximum of 0.9 to a minimum of 0.7 microgram per cc. The average $\mathrm{Cu} / \mathrm{Fe}$ ratio for the blood serum of young women is $1.15 / 1$, while that of young men appears to be $0.9 / 1$. The copper level is slightly lower in uncomplicated bilirubinemia and in untreated hypothyroidism, markedly lower in the cord blood of newly born infants, slightly higher in pernicious anemia and untreated diabetes, and markedly higher in cancer, pregnancy, leukemia, advanced tuberculosis, hyperthyroidism, and acute toxemia.

The capacity of the blood serum to inhibit the proteolytic action of trypsin is roughly proportional to the copper level. The antitrypsin content of potent extracts of the Rous chicken sarcoma becomes separated into the tumor-inhibiting fraction, whereas the metal content appears to accompany the tumor-inducing fraction upon isoelectric fractionation.

An increased $\mathrm{Cu} / \mathrm{Fe}$ ratio of the blood serum may have the same significance in man that it has in certain marine forms accustomed to live in the lower levels of the sea, namely, an increased adaptation to oxygen want. A lag in the rate at which oxygen is supplied to the metabolizing tissues over the rate at which it has tended to become consumed would appear to have been present in all of the conditions in which the elevation of the $\mathrm{Cu} / \mathrm{Fe}$ ratio was observed.

A preliminary study of oxygen want in rabbits maintained in an atmosphere containing carbon monoxide has indicated the existence of two distinct effects upon the $\mathrm{Cu} / \mathrm{Fe}$ ratio. Rabbits responding with a compensatory increase in hemoglobin production and a parallel increase in the production of intracellular enzymes (as indicated by the greatly increased copper and iron content of their serums) appear to show almost no shift in $\mathrm{Cu} / \mathrm{Fe}$ ratio. Rabbits unable to produce a compensatory increase in hemoglobin appear to show a rise in $\mathrm{Cu} / \mathrm{Fe}$ ratio comparable to that observed in cancer and in acute toxemia.

Blood Cultures of Apparently Healthy Persons. By Allan F. Reith, Рн.D., and Theodore L. Souier, M.D., Milwaukee, Wis.

Blood culture studies have been made from a series of 293 apparently healthy persons all of whom were actively engaged in their usual occupation. Each 
person was given a thorough physical examination including complete dental roentgenograms and examination of the prostate, sinuses, etc., for evidence of infection.

After careful sterilization of the skin, blood was withdrawn from the cubital vein and $5 \mathrm{cc}$. divided among three flasks of glucose brain broth to give dilutions of $1: 100,1: 300$ and $1: 500$. Cultures were examined after 7,14 , and 30 days, or as soon as growth became visible.

Bacterial growth was obtained in 113 of 293 cultures. Cultures containing streptococci, diplococci, diphtheroids, $M$. catarrhalis, $B$. coli, or obligatory anaerobic rods were considered positive. Those containing only staphylococci were considered to be questionable. All others were regarded as negative. On this basis 65 cultures were positive ( 22 per cent), 28 were questionable (10 per cent), and 200 were negative ( 68 per cent).

In the series, cultures were made from 194 persons with definite evidence of focal infection. Of these 53 (27 per cent) gave positive and 124 (64 per cent) gave negative cultures. The remaining 99 persons were without demonstrable foci of infection. Only 12 of these (12 per cent) gave positive and 76 (77 per cent) gave negative cultures.

Joint or muscle pain, including chronic infectious arthritis diagnosed clinically in 7, was present in 24 persons. Blood cultures were positive for streptococci or diplococci in 10 , or 42 per cent, of these.

A seasonal variation in the incidence of positive blood cultures from persons without demonstrable foci of infection suggests that acute respiratory infections may be responsible for some of the positive cultures.

The Intradermal Test in the Diagnosis of Tularemia. By LeE Foshay, M.D., Cincinnati, Ohio.

Intradermal injection of dilute suspensions of Pasteurella tularensis provokes a lasting unequivocal skin reaction in all patients with tularemia. The reaction is positive a week before agglutinins appear in the blood, occurring as early as the fourth day of disease. It is invariably positive in tularemia, it is never positive in normal controls, it is not positive in any other disease within the limited experience to date, it becomes negative only when convalescence is complete.

Methods are described for preparing suitable antigens.

Modifications of the reaction caused by persistent subcutaneous treatment of three cases with adequate antigens will be discussed.

\section{A New Simple Method for Determining the Efficiency of the Peripheral Arterial Circulation. By George W. Scupham, M.D., and Card Johnson, M.D., Chicago, Ill.}

The instrument used is a sensitive air plethysmometer actuating a colored droplet of alcohol. It is made in various sizes and shapes to fit the digits or other parts of both extremities. Variations in volume of a digit with each cardiac impulse may be observed and measured. This volume change is shown by the pulsation of the droplet in a graduated $1 \mathrm{cc}$. pipette.

The character of the pulsation in normals is quite uniform under uniform environmental conditions. It is diminished or absent in thromboangiitis obliterans and in arteriosclerosis depending on the severity of the occluding process.

The instrument is of diagnostic value, and useful in gauging improvement in peripheral arterial disease. It is effectively and conveniently used on small members, hand, foot, or digit where the oscillometer fails. 
There are other possibilities to its usefulness. There are increased pulsations in aortic insufficiency, heart block and in peripheral vasodilation. The increased pulsation of patients with fever is noteworthy.

A diminution in volume which occurs with respiration is interpreted to be the result of the aspiration due to inspiration and its effect on the venous return to the heart.

A method of permanently recording the movements of the droplet photographically on a moving strip of bromide paper is being devised so that accurate measurement of its oscillation and of the contour of the wave may be made.

Arteriolar Studies in Patients with Hypertensive Heart Disease without Hypertension. By Emmet F. Horine, M.D., Morris M. Weiss, M.D. (by invitation) and Marion F. BEARD, M.D. (by invitation), Louisville, Ky.

Biopsy specimens of the deltoid muscle were obtained from eleven individuals with cardiac enlargement but normal blood pressure and no other factor to explain the enlargement. Histological changes of the arterioles similar to those encountered in known hypertensives were found in all. Careful measurements of the arterioles of the specimens gave a ratio of wall to lumen from $1: 1.31$ to 1: 0.70 similar to ratios found in known hypertensives and in marked contrast to the normal ratio of $1: 2$. Three patients are now dead and one autopsy was obtained in which a generalized arteriolar sclerosis was found. The frequency with which hypertensives show arteriolar lesions of the voluntary muscles is discussed. A diagnosis of " hypertensive heart disease without hypertension" may be confirmed by biopsy studies of the voluntary muscles.

The Effects of Enclosing the Kidney in a Rigid Cast. By Samuel Soskin, M.D., and (by invitation) OTto SaPhIR, M.D., Chicago, Ill.

Kidneys were aseptically enclosed in gauze and collodium casts, care being taken to avoid interference with the blood supply or ureters. When one kidney of an animal was so treated and the other kidney removed after several days or weeks, the animal invariably died. If, however, the cast was removed from the one kidney before the other kidney was extirpated, the animal often survived. These results, together with some chemical studies, indicate that the presence of such a cast incapacitates the kidney, but leaves little permanent damage detectable by ordinary means. Those animals which do not survive die without evidence of nitrogen retention in the blood. The manner in which the cast, while in place, interferes with the kidney function, and the circumstances of the death of some of the animals after the cast is removed, are suggestive of the existence of kidney functions with which we are not familiar.

\section{Chronic Lymphatic Reaction or Atypical Leukemia, Tw' Cases of Nine and Ten Years' Duration. By Edgar T. Herrmann, M.D., St. Paul, Minn.}

Origin and function of lymphocytes-probable defense rôle-classification of lymphatic reactions (slide) -exercise lymphocytosis (slide)-five types of lymphatic reaction (slide) - two cases under discussion (slide) - case of subacute aleukemic leukemia with hemorrhagic diathesis (slide) shown in contrast to previous two cases. The cases under discussion are both over seventy years of age and for ten years have shown no splenic or glandular enlargement of any kind. Total leucocyte counts have varied between 16,000 and 25,000 with a preponderant percentage, from 60 to 80 , being lymphocytes. A study of the slides by Dr. Downey has failed to establish the diagnosis of chronic lymphatic leukemia. 
Aleukemic Myelosis with Osteosclerosis. By J. F. BREDECK, M.D., and D. H. Stephens, M.D. (introduced by David P. Barr, M.D.), St. Louis, Mo.

The report of Henck in 1879 of two cases of leukemia with bone changes has been followed by infrequent descriptions of generalized osteosclerosis of the skeleton accompanied by leukemic infiltrations of viscera with leukemoid peripheral blood picture in the presence of normal or slightly elevated total count. Two such cases in adults are described. The disease is apparently primarily an affection of bone, with compensatory myelogenous activity in extramedullary blood forming organs.

A Comparison of the Newer Methods of Testing Renal Function. By J. M. Hayman, JR., M.D., Cleveland, Ohio.

The creatinine test of Rehberg, the urea clearance test of Van Slyke, the serum sulphate level, together with the phenolsulphonphthalein excretion, concentration tests, etc., have been run on a group of normal and nephritic patients, and the results scrutinized in the light of pathological findings and, where possible, with count of the number of glomeruli in the kidneys. It is concluded that by the use of several of the tests, particularly a combination of creatinine and urea clearance, a more detailed picture of kidney function can be obtained than by any test alone.

Nature of Fibrillation and Flutter of the Heart. By L. N. KATZ, M.D., and (by invitation) W. A. Brams, M.D., Chicago, Ill.

Several theories have been advanced to explain fibrillation and flutter of the heart. The two most widely accepted theories are the single pacemaker theory and the "circus movement" theory of Lewis.

Our experiments were planned to test the validity of these theories. For this purpose, electrograms of the exposed ventricles and of the exposed auricles were made during fibrillation and flutter. The ventricles were severed during the fibrillation and it was found that the two isolated chambers continued to fibrillate at approximately the same frequency as before. Similarly, in the case of the auricle, a crushing clamp was employed to completely separate the two chambers, with the same result.

These results are incompatible with the two foregoing theories.

The Value of the Audiometer Test in Neuro-Syphilis. By Leon Bromberg, M.D., and RoBert D. SMith, M.D. (by invitation), Chicago, Ill.

Using a 2-A Westinghouse audiometer, curves were recorded on 100 unselected consecutive cases under treatment at the Public Health Institute. At the time these observations were made, the otologist was not informed about the clinical history or serologic findings of the patient. The test conditions were entirely standard for all patients, and were well controlled.

A careful review of these cases has shown a remarkable correspondence between defects in the upper vibratory tone range (1024 to 8192 double vibrations per second) and clinical or laboratory evidence of neurosyphilis. Considering the predilection which syphilis shows for the auditory nerve, the authors believe that the audiometer test offers highly useful supplementary evidence of invasion of the nervous system. The test is particularly valuable in cases where an examination of the cerebrospinal fluid is not feasible. Changes in the audiometer curve may also offer a useful index of response to treatment. The pathophysiology of the auditory defect is briefly discussed, and the authors present a few illustrative slides of characteristic audiograms and case histories. 
Organization of a Convalescent Serum Center and a Preliminary Report of Some of the Results in the Use of Several Convalescent Serums. By S. O. Levinson, M.D. (by invitation), Clarice McDougall, M.D. (by invitation), and William Thalhimer, M.D., Chicago, Ill.

A brief description of the organization of a serum center. An outline of the necessary equipment and personnel and of the cooperation necessary for securing and distributing serum from patients recovered from poliomyelitis, measles, chicken pox, scarlet fever, etc. A brief preliminary report of some of the results in prophylaxis and therapy.

Uric Acid. Excretion as Influenced by Ultraviolet Light. By M. G. PeterMAN, M.D., Milwaukee, Wis.

A group of thirty-eight convalescent children has been studied to determine the effect of sunshine, artificial quartz-light, and indoor life on uric acid excretion of these children on normal and on high purin diets. Urines were collected during these various periods and the total uric acid excretion determined. No significant variations were noted in the amount excreted under these conditions.

The Relation of the Pain of Peptic Ulcers to Gastric Acidity and Motility. By Jacob Meyer, M.D., Dorothy Fetter, M.D. (by invitation), and Alfred A. Strauss, M.D., Chicago, Ill.

Studies of epigastric pain in relation to gastric acidity and gastric motility were made in 24 patients. Palmer's acid test was used and the gastric motility was studied by the balloon method.

In eight of fifteen patients with ulcer, pain occurred in response to the injection of hydrochloric acid and had no relation to the gastric motility.

In six of the other ulcer patients, pain occurred synchronously with gastric contractions and not in response to acid stimulation.

In nine cases of epigastric pain with no demonstrable lesion of the stomach or duodenum, six reacted positively to the acid test. Pain did not occur synchronously with gastric contractions in any of these cases.

In four patients with gastric and duodenal ulcer, operation under local anaesthesia, direct application of dilute $\mathrm{HCl}$ in varying strengths, of 0.5 per cent, 1 per cent and 5 per cent resulted in negative pain response. Two of these were acid positive by the Palmer test, and two were definitely positive by the motility test.

The results indicate that hydrochloric acid is not responsible for the pain in all cases of peptic ulcer. Hunger contractions or motility is undoubtedly an equally important factor. The positive acid response in cases without intrinsic gastric and duodenal lesions, as in gallbladder disease, chronic appendicitis and gastric neurosis, would indicate the unreliability of the acid test as a diagnostic test of gastric and duodenal ulcer.

Negative Results with the Dye Method in Showing Blood Dilution. By WILliam H. Holmes, M.D., and J. R. Miller, M.D., Chicago, Ill.

Fifteen hundred cc. of 0.9 per cent sodium chloride solution were given intravenously to dogs, in which a normal blood volume value had previously been established. Blood volume determinations made immediately following the administration of fluid failed to show an increase in volume. Blood counts, hematocrit readings, and total protein determinations all indicated definite dilution. 
Chronic Idiopathic Hemoglobin Deficiency Anemia. By Charles H. Watkins, M.D., and Herbert Z. Giffin, M.D., Rochester, Minn.

This paper is chiefly a consideration of the clinical manifestations of chronic hemoglobin deficiency anemia in which a definite etiological factor has not been discovered, as exemplified in a study of more than one hundred cases. The syndrome has been described in the literature under various names-chronic chlorosis, chloranemia, hypochromic anemia with achlorhydria and simple achlorhydric anemia. Some of the clinical considerations are as follows: The occurrence of achlorhydria, the presence of acid after histamine, the number of cases that show normal acidity or hyperacidity, the occurrence of menorrhagia, glossitis, stomatitis and paresthesias. It is clearly demonstrated that the clinical syndrome of chronic idiopathic hemoglobin deficiency anemia is not necessarily accompanied by achlorhydria.

Blood Glucose Clearance: Determination by a Microinterval Technique. By Richard M. McKean, M.D., Detroit, Mich.

Short-interval blood sugar studies were carried out on 100 normal subjects, mild diabetics, severe diabetics, and individuals suffering from hyperthyroidism, following the intravenous injection of 0.2 gram of glucose per kilogram body weight. They showed that the maximum blood sugar levels were reached within 3 to 7 minutes, and the 15-minute curve of assimilation was similar to that observed in the 3-hour period following oral administration of glucose. This method offers certain advantages over the oral method of glucose tolerance determination, chiefly in the amount of glucose administered, time required for the test, elimination of the enteral absorption factor, and added comparability of resultant curves.

Eosinophilia Due to the Administration of Digitalis. By Abigail E. Smith, M.D., and Stanley R. Benner, M.D. (introduced by D. P. Barr, M.D.), St. Louis, Mo.

A case of decompensated heart disease being treated with digitalis showed an eosinophilia of 25 per cent during administration of the drug. While the drug was withheld for 3 weeks the eosinophile count dropped gradually to 11 per cent. Upon resuming digitalis administration the percentage of eosinophiles rose promptly to 30 per cent. References to "digitalis eosinophilia" are found in the foreign literature. The phenomenon is discussed as a possible effect of vagus stimulation by the drug digitalis.

Incidence of Organic and Functional Disease in Patients Whose Symptoms are Chiefly Abdominal. By S. F. Adams, M.D., Rochester, Minn.

This study was undertaken in order to find out how many people with "stomach trouble" as their chief complaint had organic disease.

These people were sufficiently concerned about their trouble to travel from three blocks to three thousand miles for advice. None of these people was referred by a physician.

The preliminary survey showed that 39 per cent had organic disease and that 52 per cent had no lesion of the stomach, biliary tract, pancreas, or bowel. A few were undiagnosed; there were 9 per cent in this so-called "indeterminate" group.

Further Experience with Hyperinsulinism. By Frank N. Allan, M.D., Rochester, Minn.

At the meeting of the Central Society for Clinical Research in 1928, observations on three cases of hyperinsulinism were reported. Five additional 
cases have since been studied. A review is given of investigations in the series of eight cases. The diagnosis is discussed from clinical and laboratory standpoints. In seven cases operation was performed. The results of medical and surgical treatment are presented.

Prolonged Treatment of Polycythemia Vera with Phenylhydrazine. By EDGAR V. Allen, M.D., and Herbert Z. Giffin, M.D., Rochester, Minn.

Detailed records are available regarding eleven patients with polycythemia vera who have been treated with phenylhydrazine since 1925 . Survey of these data shows that in most instances the erythrocyte count can be maintained at a nearly normal level with small amounts of phenylhydrazine taken two or three times a week, or one day out of each week.

In three instances the patients had some difficulty in regulating the dosage with the result that the erythrocytes were occasionally too high or too low.

Four patients have been able to dispense with the use of phenylhydrazine with no return of the polycythemia after they have used the drug in the manner indicated above for periods of two or three years. The remaining patients have found it necessary to continue with the phenylhydrazine but have controlled the polycythemia satisfactorily from the standpoint of the number of erythrocytes and the symptoms usually associated with this disease.

There is no evidence from a clinical standpoint that the prolonged administration of phenylhydrazine does any damage to the organs of the body, but laboratory records were not available.

There is no evidence from our study that patients acquire a tolerance to phenylhydrazine; increasing doses of the drug are not necessary.

Lipoid Nephrosis and the Nephrotic Syndrome. By E. G. BAnnick, M.D., Rochester, Minn.

This report does not embrace a review of the literature on this subject since this has been presented repeatedly and quite recently. It is a summary of the author's personal experience with this problem together with an analysis of practically all the adult patients presenting the nephrotic syndrome that have been studied at the Mayo Clinic between the years 1921 and 1930, inclusively. In most instances a fairly satisfactory follow-up record has been obtained.

Special necropsy studies have been made in conjunction with $\mathrm{Dr}$. N. W. Barker and Dr. D. L. Wilbur on the patients in this group that died at the clinic during this period. None of the necropsy cases represent the primary uncomplicated form of lipoid nephrosis in its strictest analysis, and yet in several instances the evidence of associated nephritis was so slight that very careful study was necessary to detect it. In these cases the pathological findings were practically identical with those of lipoid nephrosis that have been reported in the literature. The slight changes noted in the glomeruli, even in this group and by special stains, were not sufficient to warrant the assumption that so-called lipoid nephrosis is a form or stage of glomerulonephritis. However, the not infrequent association of the nephrotic syndrome with true glomerulonephritis, and the fact that the kidney even in pure lipoid nephrosis shows the same pathological picture and is the only organ which is consistently involved, serve to prevent divorcing lipoid nephrosis entirely from renal diseases and considering it a general metabolic disturbance. On the other hand, the extra-renal factors are so pronounced that pathological studies of the kidneys have thus far failed to offer a satisfactory solution to the problem.

From a clinical standpoint also, uncomplicated lipoid nephrosis is rare, but there is not the paucity of cases for study that the pathologists note. At least 
thirty cases have been studied at the Mayo Clinic during the years mentioned and have been so classified only after close analysis and strict adherence to the criteria that have been generally accepted. Most of these cases have subsequently improved and several have made complete recovery. A considerable number of borderline cases have been excluded, perhaps unnecessarily, because of a slight deviation from the normal criteria which have been rather arbitrarily established. The more evidence there is pointing to an associated glomerulonephritis, the poorer the prognosis becomes.

Skin Tests in Chronic Ulcerative Colitis. By J. C. T. Rogers, M.D. (by invitation) and J. Arnold Bargen, M.D., Rochester, Minn.

To a series of normal controls were administered antigens prepared from the diplostreptococcus of chronic ulcerative colitis, streptococcus fecalis and the enterococcus by intradermal injection. Another series of fifty-six unselected patients with chronic ulcerative colitis were injected with the same antigens, intradermally, and the results recorded at intervals. Positive skin tests became negative during the administration of the specific antibody solution (concentrated serum) and coincident with the clinical relief of symptoms of colitis.

The Tabacco Factor in Thrombo-Angiitis Obliterans. By NeLson W. BARKER, M.D., Rochester, Minn.

A study of the consumption of tobacco in 350 cases of thrombo-angiitis obliterans as compared with that in 350 control cases disclosed that there were many more heavy smokers in the first group than in the second. However, the small but definite number of cases of thrombo-angiitis obliterans in which the patients had never used tobacco or used only small amounts, raises the question of whether tobacco may be a direct etiologic factor in the disease. There is some statistical evidence to show that the disease tends to be more malignant if patients are heavy smokers, and that recurrences are fewer if the consumption of tobacco is decreased or stopped.

On the Pathogenesis of Angina Pectoris with Special Reference to Its Association with Certain Severe Anemias. Report of a Case of Angina Resulting from Severe Posthemorrhagic Anemia. By Moses Barron, M.D., Minneapolis, Minn.

Exact pathogenesis of angina pectoris is still undetermined. Albutt's aortic hypothesis is falling into discard. Jenner's coronary basis is most likely the correct one. The anatomical basis; postmortem findings. Experimental support for coronary narrowing by Sutton and Lueth. Coronary spasm in ischemia. Anemia of muscle is the cause. Wenckebach's recent hypothesis of thinning and distension of muscular coats in arteriosclerosis. Angina pectoris is occasionally associated with severe anemias as reported by Herrick, Willius and Griffin, and others.

In all available literature no case is reported of angina associated with acute posthemorrhagic anemia. All anemias in the reported cases, whether pernicious or secondary in type, are chronic.

In this study the angina followed immediately upon a severe gastrointestinal hemorrhage.

Detailed report is given of a case with blood studies and electrocardiagrams. With the disappearance of the anemia, the angina disappeared and the patient returned to his usual occupation. 
An Inorganic Standard for Hemoglobin Determininations. By A. P. BRIGGS, M.D., St. Louis, Mo.

A solution which matches the acid hematin solution ordinarily used for colorimetric determination of hemoglobin has been prepared by adding small quantities of copper sulfate, chromium sulfate, and cobalt sulfate to a solution of ferric sulfate. The color matches acid hematin somewhat more closely than the Newcomer disc, has a known chemical composition, and its color value is permanent. Compared with acid hematin with the aid of a spectrophotometer roughly parallel extinction coefficient curves were obtained. The solution has been standardized by comparison with the acid hematin color of various bloods, the hemoglobin content of which had been calculated from iron determinations.

Acidity Patterns of Gastric Juice Obtained by Continuous Aspiration. By Charles L. Brown, M.D., and Friedrich Engelbach, M.D. (by invitation), Ann Arbor, Mich.

A method of continuous aspiration of gastric contents is described. Histamine was administered but no test meal was given. These studies are presented under two headings: (1) The acidity pattern of the gastric juice obtained from the same individual (normal) under similar experimental circumstances on different days. Ten or more observations are made in each case. It was found that the acidity curve may vary considerably in some respects at times. In general, however, the peak of the acidity curve was reached essentially at the same time after histamine administration during each observation. (2) The acidity pattern of the gastric juice obtained from the same individual on different days, but having graded doses of histamine. From these studies it would seem that the usual dose of histamine may be larger than necessary.

Nutrition of Sick Children. By M. G. Peterman, M.D., Milwaukee, Wis.

This investigation was a study to determine the food requirements of children sick in the hospital with chronic diseases, particularly tuberculous bone lesions. A particular interest was in the effect of diets with high fat, acid ash, or basic ash, and the influence of $\mathrm{NaCl}$. Twelve children were studied for a period of nine months. All of the necessary procedures such as physical examination, blood counts, urinalysis, etc., were made. The patients were weighed and measured once a week.

The Sauerbruch-Hermannsdorfer diet has been recommended for tuberculous patients. This diet was reported to be a basic ash (but actually found to be an acid ash), low $\mathrm{NaCl}$, high fat, high mineral, and high vitamin feeding. There was no striking clinical improvement in our study in any one period on any particular diet. The food requirements were found to be approximately those for normal, well, active children. From our study it appeared that the ideal diet for sick children was a basic ash, high vitamin, high mineral, attractive diet.

The Vasodilating Effects of Alcohol. By George E. Brown, M.D., and EDWARD N. CooK, M.D. (by invitation), Rochester, Minn.

Various drugs have been tested for their vasodilating effect on the peripheral arteries. The most effective of them has been ethyl alcohol administered by mouth; this produces well-defined, prolonged vasodilation as measured by the surface temperature and the elimination of heat. Its action in a high percentage of cases of arteriosclerosis shows sharp vasodilation in the affected extremities. 
A Study of Proportional Heart Weights in Human Subjects. By JAmes G. CARR, M.D., and Victor Levine, M.D. (by invitation) Chicago, Ill.

Hermann in 1925, on the basis of extensive experimental work, recommended a method for the preparation of hearts and the separation of the ventricles preliminary to weighing the ventricles. Following Hermann's method fifty human hearts have been prepared and weighed. This group includes hearts which are normal and hearts representative of the common varieties of cardiac disease. The relationships of the ventricles to each other, to the total heart weight and to the body weight have been determined and are presented.

The Coexistence of Pernicious Anemia and Carcinoma of the Stomach. By H. Milton Conner, M.D., Rochester, Minn., and Ivar W. Birkeland, M.D. (by invitation), Seattle, Wash.

Eighteen cases are reported from the Mayo Clinic in which there arose the question of the coexistence of carcinoma of the stomach and pernicious anemia. In seven cases, the diagnosis of both pernicious anemia and carcinoma of the stomach in the same individual seemed reasonably certain; in five cases, the existence of carcinoma of the stomach seemed practically sure and that of pernicious anemia probable; in two cases, the diagnosis of carcinoma of the stomach depended almost entirely on roentgenologic examination, but the existence of pernicious anemia was probable; in four cases, the existence of carcinoma was reasonably certain and pernicious anemia was possible.

Extract of Livers of Codfish in the Treatment of Pernicious Anemia. By $\mathrm{H}$. Milton Conner, M.D., Rochester, Minn.

Hansen and Stubb used livers of codfish in the treatment of pernicious anemia in five cases with good results. An extract was not so efficacious.

Connery employed an aqueous extract of livers of codfish in six cases with an effect comparable to that obtained with mammalian liver and its extract.

In this study an aqueous extract of codfish livers has been given to ten patients, two of whom had the liver extract only a few days because of intolerance, and one of whom was ambulant. Seven patients were studied in the hospital.

In an average of twenty-seven and six-tenths days in the hospital, the average of the erythrocytes of these seven patients increased from $1,710,000$ to $3,390,000$, the hemoglobin from 39.7 per cent to 67.6 per cent, and the leucocytes from 5,200 to 7,800. The increase in erythrocytes averaged 426,000 a week. The reticulocytes rose in one case to 30.4 per cent and the average height of the peak of the reticulocyte curve was 12 per cent of the total number of erythrocytes. The general condition was equally improved. Too few patients were observed to justify any conclusions regarding the effect on the nervous system, although there was some subjective improvement of three of the four patients who had subacute combined degeneration of the spinal cord, but this improvement was not marked during the short stay in the hospital.

Some Qualitative Differences in the Action of Certain Commercial Digitalis Preparations. By G. K. Fenn, M.D., and N. C. Gilbert, M.D., Chicago, IIl.

In the course of an investigation on the effect of digitalis upon the coronary flow, it was observed that in addition to a difference in action on the coronary arteries, there was also a difference in the action of certain preparations on the conducting tissues, upon muscular irritability, and in the mode of death. 
These points were worked out in a separate series of animals and are reported upon.

A Study of the Effect of Sodium Fluoride on the Dog's Thyroid. By Leo Gottleib, M.D. (by invitation), and Samuel B. Grant, M.D., St. Louis, Mo.

A relatively small number of dogs, with one lobe of the thyroid removed from each dog as control gland tissue, were given sodium fluoride either by mouth or intravenously, and the remaining lobe of the thyroid removed after various intervals and various dosages. The iodine content of the control gland and the gland after administration of fluoride showed no constant change, and there were no gross nor microscopic alterations in the structure of the gland.

L. Goldemberg and others have been able to produce goiters and a condition resembling myxedema in sheep, guinea pigs, etc. Goldemberg has also used fluorine in the treatment of exophthalmic goiter.

Changes in Haemodynamics under Physiological and Pathological Conditions. By W. F. Hamilton, M.D., J. W. Moore, M.D., and J. M. Kinsman, M.D., Louisville, Ky.

Various conditions which result in an increase in stroke volume of normal men and animals cause also a marked and quantitatively measurable increase in the intrathoracic blood volume. This is brought about through the increased left ventricular filling pressure on the elastic lung capillaries.

If the left ventricle is weakened through disease (congestive failure) an increased filling pressure is necessary to get a more or less adequate stroke volume (Starling's law of the heart). This increase in left ventricular filling pressure results in a marked and quantitatively measurable engorgement of the intrathoracic blood spaces. The stroke volume and the intrathoracic blood may, either one, approach the normal but the relation between them is strikingly different from the normal.

On recovery from decompensation the greatest and most constant change is a movement toward normal of the ratio between stroke volume and heart-lung blood volume. The stroke volume, the minute volume and the heart-lung blood volume may increase, decrease or remain unchanged.

The mean circulation time,- the time from the middle of the injection period to the center of gravity of the time concentration curve-is always increased in congestive heart failure, returning toward normal on compensation.

The total blood volume (vital red method) is almost uniformly increased by twenty per cent or more in congestive heart failure.

These considerations are illustrated by spot diagrams embodying the results of 100 experiments on man and 50 experiments on the dog.

The Occurrence of Free Hydrochloric Acid in the Gastric Content of Patients with a Macrocytic Hyperchromatic Type of Anemia. By FrAN K J. HECK, M.D., Rochester, Minn.

The occurrence of cases of macrocytic hyperchromatic anemias, other than pernicious anemia, in this country is unusual. The two cases reported here both were men, one aged 25 and the other aged 60 . In both cases there was a severe degree of anemia with an average red count of 2,000,000. Test meal analysis showed the presence of normal amounts of free hydrochloric acid. In the first case there was rapid improvement when the patient was treated with six tubes of Lilly's liver extract per day. A reticulated erythrocyte response similar to that of pernicious anemia was seen in this case. Within five weeks' 
time the blood count had returned practically to normal. At the end of one year the blood count was normal, although the patient was taking only one tube of Lilly's liver extract per day. In the second case, on mixed treatment, using iron and liver or liver extract there was definite improvement. However, as soon as the liver or liver extract was discontinued there was a relapse both clinically and from the standpoint of blood count.

Morphologically these two cases showed all the findings common to pernicious anemia and it is important to point out that it is necessary to have other corroborative evidence for a positive diagnosis of pernicious anemia.

Thrombo-Angiitis Obliterans in Women. By BayARd T. Horton, M.D., and

George E. Brown, M.D., Rochester, Minn.

Thrombo-angiitis obliterans is an occlusive vascular disease which usually affects men between the ages of twenty and forty-five years. The literature contains two acceptable reports of cases of this disease in females. Although more than 600 cases of thrombo-angiitis obliterans have been observed at the Mayo Clinic the present report of eight cases is the first series of cases of women to be put on record; the diagnosis in two of these cases was proved pathologically. We know of no other disease in which common tissues are involved, such as veins and arteries, in which the disease is so nearly exclusively confined to one sex. A satisfactory explanation of sex distribution in this disease has not been determined.

Effect of Liver Extract Intravenously on the Blood of Patients with Pernicious Anemia. By Raphael Isaacs, M.D., S. Milton Goldhamer, M.D. (by invitation) and Cyrus C. STURgis, M.D., Ann Arbor, Mich.

Liver extract was given intravenously to two patients and four-hourly blood studies were made throughout the day and night for eleven days. The first evidence of a reticulocyte increase was in 28 to 43 hours; the calculated maximum reticulocyte percentage (on the basis of the oral method) was reached in 68 hours, the actual maximum in 88 to 108 hours; the return to normal was in 20 days after one dose. Although the dosage was 0.1 gram per kilogram body weight (total 6 grams in one patient, 10 grams in the other), the maximum reticulocyte rise was greater than the calculated response for 6 vials (27 grams) of liver extract daily by mouth or, for the period studied, 297 grams. The symptomatic improvement was very prompt and did not differ from the usual response to liver extract or ventriculin by mouth. These results were confirmed by 84 subsequent intravenous injections in 24 patients with pernicious anemia.

Studies on Mercurial Diuresis: The Effect of Ephedrine in Enhancing the Action of Salyrgan. By CarL A. Johnson, M.D., and R. H. Young, M.D. (by invitation), Chicago, Ill.

The authors have attempted to show by accurate animal experiments that ephedrine enhances the action of salyrgan by increasing the blood supply to the kidney through vasodilation of the kidney vessels and increased cardiac output.

Dogs anesthetised with $\mathrm{Na}$ barbital were used throughout the work. Both ureters were cannulated and simultaneous records of blood pressure and urine flow were made.

Ephedrine was injected at various intervals before and after the salyrgan injection. The optimum time for injection of ephedrine to promote maximum diuresis was about one-half hour after the salyrgan injection. Under these 
conditions ephedrine increased the urinary output following the salyrgan from about $8 \mathrm{cc}$. per half hour to $40 \mathrm{cc}$. per half hour.

The authors feel that this method of inducing diuresis may be valuable in overcoming the reflex anuria often seen clinically.

Clinical Observations on Oxygen Therapy in Heart Disease. By W. W. HAMBURGER, M.D., L. N. KATZ, M.D., and (by invitation) S. H. RUBINFELD, M.D., Chicago, Ill.

Six patients with heart disease were treated with 40 per cent oxygen eight times with clinical improvement three times. Two of these patients had severe acute anoxemia associated with heart failure. Clinical improvement occurred twice in three oxygen treatments. One good result was obtained out of five treatments in the four cardiac patients with long standing heart insufficiency and mild anoxemia. One of the patients died in the oxygen room, and another soon after removal from the oxygen tent. No striking diuretic action was noted such as reported by Barach.

The most constant changes, aside from the alteration of the composition of the arterial blood, noted in the patients while in the oxygen-rich environment, were a slowing in heart rate and a diminution in the minute volume of respiration. These changes also occurred in the control and non-cardiac cases studied.

Absorption from the Pleural Cavity of Dogs. II. The Lymphatic System. By Willis S. Lemon, M.D., and (by invitation) George M. Higgins, M.D., Rochester, Minn.

A graphite preparation was injected into the pleural space of dogs. This provided a means for the study of the functional lymphatic system of the dog's thorax.

The lymph nodes arrange themselves naturally into two main groups: parietal and visceral. The parietal group comprises the sternal, the vertebral, and the diaphragmatic nodes; the visceral group includes the mediastinal, pulmonary, tracheobronchial and tracheal nodes.

The lymph drainage associated with these nodes may be grouped into six routes, two of which conduct lymph caudad and four cephalad. On the dorsal wall of the thorax lymph vessels pass caudad, and empty into a large lymph node which lies dorsal to the anterior pole of each kidney. Efferent vessels from these nodes lead directly into the cisterna chyli. The cephalad lymph drainage along the dorsal median line is less easily demonstrated than the caudad one. Intercostal segmental collateral vessels are not constant, although they are far more nearly constant on the right than on the left side. On the right the collecting channels empty into the caudad tracheal group of nodes; on the left they empty into the mediastinal group of nodes.

On each side of the sternum the lymphatic drainage system from the ventral pleura comprises a series of segmental channels which converge to form an extensive plexus lateral to the internal thoracic blood vessels beneath the transverse thoracic muscle. Both pleural and abdominal lymph, draining the plexus, empty into the sternal group of nodes at the level of the first and second interspaces. The terminal efferent vessels from the sternal and the tracheal nodes usually become confluent and together join the thoracic duct or the right lymphatic duct.

The Effect of Ischemia in Different Parts of the Body on Blood Pressure. By A. J. Miller, M.D., Louisville, Ky.

The report consists of a discussion of the findings in experiments on animals in which the blood supply was partly shut off to the brain, voluntary muscles, 
kidneys and other viscera, and the immediate and remote effects on blood pressure observed.

Scarlet Fever Streptococcus Antitoxin: Its Use in the Treatment of Scarlet Fever. By A. Graeme Mitchell, M.D., and (by invitation) Frank E. Stevenson, M.D., and M. V. Veldee, M.D., Cincinnati, Ohio.

The purpose of this study was to determine, if possible, the effect of scarlet fever antitoxin in treatment of an unselected group of cases. In order to do this, every other patient suffering from scarlet fever admitted to the Contagious Wards of the Cincinnati General Hospital was given antitoxin. That is, no selection was made according to the severity of the case. Complete protocols were kept and the analysis of these was made without a knowledge at the time of the method of treatment of the patient. The study will be presented by a lantern slide demonstration. In so far as this group of patients was concerned the study seemed to show that the antitoxin had a specific neutralizing effect because there was decrease in the duration of the rash, a change in the character and extent of the desquamation, and a reduction in the number of complications. For example, the number of complications was diminished in the serum-treated group in relation to the expected number of complications as judged by their frequency in the control group. There was a large percentage of serum sickness but this occurred twice as frequently in patients who had previously received injections of horse serum in some form.

Observations on the Urinary Cast Count During Administration of Salyrgan. By Charles L. Brown, M.D., and (by invitation) Friedrich ENGelBACH, M.D., Ann Arbor, Mich.

In recent years certain mercurial compounds have been used rather widely as diuretics. The question is raised as to whether these substances in clinical doses are harmful to the kidneys. The urinary cast count offers a possible index to nephrotoxicity. This type of observation was carried out during the administration of salyrgan as follows: (1) to normal rats in graded doses; (2) in clinical doses to cases of cirrhosis of liver, with ascites but no evidence of renal disease, and (3) in clinical doses to one case of subacute nephritis with edema (nephrosis ?). In the rat, salyrgan in the dose corresponding to the human clinical dose caused no appreciable change in the urinary cast output. Larger doses may cause an increase in the cast output, which tends to be temporary, if the dose is not too large. In some of the cirrhosis cases there was slight increase in the cast count which tended to be temporary. In the case of nephritis the cast output was not changed appreciably.

Essential (Primary) Hypertension: A Clinical and Morphological Study of 375 Cases. By Franc.Is D. MUrPhy, M.D., and (by invitation) JoHN GrILl, M.D., Milwaukee, Wis.

Essential (primary) hypertension may be associated with such a variety of clinical and morphological changes that successive stages of this disorder may appear to be separate diseases. From a clinical and histological study of 375 cases of essential hypertension an attempt is made to show that the various syndromes and vascular lesions observed are manifestations of the same process varying in its intensity and its speed of advancement. The cases are arranged into three groups representing three consecutives stages of the disease. In group 1 are the earliest cases illustrating the functional stage, in which symptoms are unrelated to vascular lesions. An accident or an infection caused death in this group before arteriosclerosis became well established. 
Group 2, or the arteriosclerotic stage embraces the cases in a more advanced stage in which arteriosclerosis is a prominent feature; death resulted usually from arteriosclerotic lesions.

Finally, group 3 includes the cases with a rapid and stormy course ending fatally within a short time. Histologically, these cases are distinguished by advanced arteriosclerosis with necrotic lesions of the arterioles of the kidney and other organs. Group 3 may be classed as the arteriolonecrotic group. Group 1 and 2 are commonly designated as "benign" hypertension and group 3 as " malignant" hypertension.

There are 43 cases in group 1, 303 in group 2, and 29 in group 3. A statistical analysis is given in tabular form of the causes of death, complications, heart weights, and age incidence in each group.

Histologically, minimal vascular changes are observed in group 1, the functional group. In group 2, there is a decided arteriosclerosis in medium sized arteries, while in smaller arteries there is a localized arteriosclerosis leading to ischemia and slow atrophy of one or other organ. The arteriosclerosis in group 3 is more diffuse and intense involving more definitely the smaller and smallest arteries and leading to necrotic lesions of the afferent glomerular arterioles as well as of arterioles in other organs.

Although, clinically, the chief features of group 3 are fairly uniform and constant, histologically the arterial lesions, especially in the kidney, may vary considerably. Whether the renal lesions represent the results of a toxic or inflammatory influence added to a kidney already damaged by arteriosclerosis, or whether they indicate the effects of arteriosclerosis alone is a disputed question. The interpretation of these lesions and their correlation with the clinical picture are presented. It is concluded that there is no fundamental difference between the three groups, but that the numerous changes observed represent various degrees of intensity of the arteriosclerotic process.

The Diagnosis of Early Tuberculosis in the Strict Sense of the Word. By J. A. Myers, M.D., Minneapolis, Minn.

This paper is based upon results of tuberculin testing and making $\mathrm{x}$-ray films of the chests of students of nursing and medicine. On admission to such schools approximately 35 per cent react positively to the tuberculin test. After taking services in tuberculosis the incidence of positive reactors increases markedly. Along with this increase a considerable number of those who previously reacted negatively to the test but became positive during or after tuberculosis service have developed demonstrable tuberculous lesions in the lung parenchyma and cervical lymph nodes. Pleurisy with effusion is a rather common finding. In this group of students we can speak of early diagnosis in the strict sense of the word. Not one of these young adults has developed acute rapidly progressing tuberculosis but all have reacted to the infection and the disease in about the same manner as children who first become infected. They respond well to treatment. Without the tuberculin test and the $\mathrm{x}$-ray film examination most of these cases would not have been diagnosed. In other words, the lesions are too nearly minimal to be detected by our other methods of examination. This study brings to light the fact that in our schools of medicine and nursing we are transmitting tubercle bacilli to the majority of the students who have never before been infected. An alarmingly high percentage fall ill with the disease.

The Rôle of Hemolytic Streptococci in Arthritis. By I. PILot, M.D., Chicago, III.

Evidence is introduced that arthritis may be due to hemolytic streptococci of various types, the Streptococcus scarlatinae, Streptococcus erysipelatis, and 
the Streptococcus epidemicus of septic sore throat. Arthritis indistinguishable from acute rheumatic fever may develop during the third or fourth week after the onset of a streptococcus sore-throat. This interval suggests the development of an allergic state to the streptococcus. For this conception some experimental work is reported. Rabbits receiving 0.01 cc. of living Streptococcus epidemicus at weekly intervals did not develop arthritis until the third or fourth injection. Broth filtrates and vaccines apparently did not sensitize the rabbits to arthritis from minute doses of living streptococci. In the human subject the sensitizing streptococci are derived from the focus in the throat, which in our cases yielded the streptococci in swab cultures from the tonsils. The Streptococcus epidemicus can be readily differentiated from other hemolytic streptococci and the appearance and disappearance of this organism can be correlated with the arthritic manifestations. The removal of tonsils harboring this streptococcus often led to relief of arthritic symptoms.

Some Observations of the Effects of Hog Stomach Extract (Ventriculin) on Gastric Secretion and Gastro-Intestinal Symptoms. By LeoN SchIFF, M.D., and (by invitation) George Burger, M.D., and ToBa Tuhl, Chicago, Ill.

The effects of the administration of ventriculin on the symptoms of a number of patients suffering with achlorhydria were noted. Repeated determinations were made of the acidity and pepsin content of the gastric juice in these individuals.

The effects on the gastric juice of the administration of a single dose of liquid extract of ventriculin were also noted in healthy individuals as well as in those suffering with gastro-intestinal disturbances. These effects were compared with those obtained with the alcohol meal, oatmeal gruel, and following the ingestion of histamine.

The Use of Follicular Hormone in Involutional States. By Elmer L. SEvringHAUS, M.D., Madison, Wis.

The manifestations of the menopause include not only the well known vasomotor symptoms but also some features which are classed as neurological or psychiatric. In a group of 30 cases with various aspects of the climacteric 14 reported paresthesias as troublesome. In 17 cases insomnia was complained of, with no other evident explanation. Half of the series had definite psychotic symptoms, which varied from increasing nervousness to the typical involutional syndrome including despondency, subjective inadequacy for the usual tasks, emotional lability, suspicious attitude, and suicidal tendencies. These cases were seen early in the course of the mental difficulty. Of the group of 15 there were 13 who received follicular hormone as the chief material in treatment. In two cases the improvement was questionable, but in 11 there was no doubt of marked relief within a few days. Most of these cases were observed for a long enough period to give assurance of maintained help by the hormone therapy. The paresthesias and insomnia have been similarly benefited.

The frequency of obesity in the group of 30 is low, only 11 cases. Several of the obese patients had been overweight for many years. Obesity does not seem to be more frequent in the older members of this group. Hypertension was found in only 10 of the cases. The typical hot flashes were seen in 27 patients, and in two others the vasomotor instability was reported as chills of short duration.

The age range is from 32 to 56 years. The 10 cases with age 41 or less include 6 with artificial menopause, 1 with a pseudo-thyrotoxic picture, and 3 
which are apparently cases of hypofunction of the ovaries. In 2 of these 3 the use of follicular hormone has re-established regular menstrual cycles and given relief from the premature involutional psychosis which was the chief complaint.

The dose range of hormone which is effective is from 10 to 40 units daily by hypodermic route, or from 25 to 50 units each night by vaginal pessary. The commonest dose has been 20 units daily, given in one injection. Patients make the injections readily, using amniotin or theelin.

Experimental Treatment, with Stramonium, of Patients Exhibiting Evidences of Thyrotoxicosis. By R. L. SENSENICH, M.D., South Bend, Ind.

The favorable effect of atropine upon patients with exophthalmic goiter has been reported by many observers.

Stramonium was suggested for this study because of the freedom from objectionable effects when given in very large doses over long periods of time in certain other conditions, notably the sequellae of encephalitis.

The drug was given to eight thyrotoxic patients, none of the class of adenomas with hyperthyroidism. Basal metabolism readings, before treatment, ranged from plus twenty-two per cent to plus fifty-eight per cent. All had lost weight and exhibited tachycardia and marked tremor, with other diagnostic evidence of toxic thyroid states. Seven of these patients were markedly improved, metabolic rates being reduced and gains in weight being recorded. Nervousness was relieved and tremor was diminished or controlled. Heart action was slowed to some extent (eighty to ninety in recumbent rest), but not controlled to a degree comparable to the improvement otherwise attained. One patient in the menopause exhibited such unpleasant cutaneous "flush" when the drug was administered that it was impractical to continue. Some described subjective eye symptoms if they read long, but there was no evidence of increased intra-ocular tension.

The stramonium was given in the form of a preparation of the leaves, in doses varying from $71 / 2$ to 15 grains daily. No other medication was given. No bad effects were observed during more than two months of continuous administration in maximum dosage.

Summary. Improvement was observed in thyrotoxic patients, without bed rest, under stramonium treatment. This improved state was maintained throughout a period of four months following cessation of treatment. Some acceleration of the pulse rate continued, although diminished in frequency under the treatment and followed by gradual slowing after its discontinuance. Judging from reports of results obtained under atropine treatment, the use of stramonium seemed to possess some advantages in results and possibility of continuous administration in effective dosage.

This series of cases is too limited to report for the purpose of arriving at any conclusions and is given only as work in progress. The study is being continued with the thought of using stramonium in conjunction with other measures.

The Endogenous Uric Acid Metabolism in Polycythemia Vera. By S. A. Shelburne, M.D., Dallas, Texas, and (by invitation) R. F. Hanzal, M.D., Cleveland, Ohio.

The authors have measured the daily endogenous uric acid excretion in a case of polycythemia vera daily during a period of four months. These findings have been correlated with the clinical condition of the patient, the changes 
in the blood cells and the blood chemistry before, during and after two periods of treatment with phenylhydrazine.

This patient had a persistently great increase in the endogenous uric acid excretion, which is the condition one would expect if this disease is due to an increased formation of red blood cells. It is well known that increased erythrocyte formation is associated with increased uric acid excretion. We also observed some changes that indicate that phenylhydrazine has a depressing action on erythrogenesis.

Available Carbohydrate and the Blood Sugar Level in the Treatment of Older Diabetics with Cardio-Vascular Disease. By Solomon Strouse, M.D., Samuel Soskin, M.D., Louis N. Katz, M.D., and (by invitation) S. H. Rubinfeld, M.D., Chicago, Ill.

There have been many reports upon the deleterious effects of insulin shock upon the myocardium. It is not known whether the harmful action is due to the hypoglycemia or to a direct action of the insulin, or a component, upon the heart muscle. Nor is it known whether the continued administration of insulin as a therapeutic procedure, in doses small enough to avoid hypoglycemia, carries with it a similar menace to the cardiovascular system of older diabetics with cardiovascular disease. Our results show that the therapeutic use of insulin, in such patients, may be accompanied by significant cardiovascular injury. They further indicate that the blood sugar level and the carbohydrate available to the damaged myocardium are important factors in this connection.

Specificity of Certain Strains of Streptococci for Endocardium. By THEOdoRE L. Squier, M.D., and (by invitation) Norbert Enzer, M.D., and Allan F. REITH, Ph.D., Milwaukee, Wis.

Certain strains of green producing streptococci produced endocardial lesions in a high percentage of rabbits after intravenous injection. Other strains apparently culturally similar consistently failed to do so.

During the past six years 539 rabbits have been injected intravenously with 147 strains of streptococci isolated from various foci of infection. With 25 of these strains, gross heart lesions developed in one or more rabbits after intravenous injection, and were found in 79 of a total of 164 rabbits thus injected. The remaining 123 strains failed to produce heart lesions in any of 375 rabbits injected.

The production of endocardial lesions by two strains of the green producing streptococci has been demonstrated through four and seven animal passages respectively. With each passage the streptococci were recovered from the heart blood, cultured in glucose brain broth and re-injected.

With one strain isolated from the blood culture of a patient with subacute bacterial endocarditis, endocardial lesions have been produced from subcultures after various time intervals. Finally after thirteen weekly blood agar transplants, single cells were isolated and subcultures from each of three cells produced typical vegetative endocarditis.

A Clinical and Roentgenological Study of the Significance of Palpability of the Liver. By Carroll W. Osgood, M.D., and J. E. HabBe, M.D. (introduced by T. L. Squier, M.D.), Milwaukee, Wis.

Fifty-three patients without clinical evidence of hepatic disease in whom the liver was found to be palpable, were studied in comparison with a control group of fifty-three patients with non-palpable livers. Roentgenological determinations were made of the size of the right lobe and the relation of the inferior 
border of the liver to the costal margin. The level of the liver border was also determined by palpation, and observations made of the thickness and relaxation of the abdominal wall, the depth of inspiration, and the weight and build of the subject. Liver function was determined by intravenous dye tests.

Little correlation was found between palpability and liver size. The average $\mathrm{x}$-ray size of the palpable livers was no greater than that of the non-palpable ones in the control group. Practically all of the livers observed descended below the level of the costal margin with deep inspiration, and the non-palpable ones moved downward about as far as did the palpable ones. None of the livers studied exceeded the statistical limits of normal. While obviously such factors as the size of the liver, its relation to the costal margin and the tenseness of the abdominal wall, must in some instances affect palpability, no important influence was noted in this study. In the palpable group no appreciable impairment of liver function could be demonstrated by intravenous dye tests. Possible differences in firmness between the livers of the two groups could not be observed but the results obtained suggest that palpable livers are not necessarily pathological.

Gastric Achylia (Neutral Red Studies). By M. H. Streicher, M.D., Chicago, Ill.

In recent years it has been claimed by some investigators that neutral red possesses a property similar to that of histamine as a stimulant of gastric acidity. Others considered neutral red of value in the differential diagnosis between true and false achylia.

We have attempted in this work to study the function of neutral red in gastric acidity determinations. For this purpose we obtained a series of ninety-six cases and classified them into three groups based largely on time of appearance of the dye in gastric contents. Gastric contents were collected every fifteen minutes for two hours and studied.

Briefly our results show that neutral red may be used to advantage as a test of gastric function and that neutral red possesses little or no property as a stimulant of gastric acidity. In differentiating between false and true achylia it may be employed because a prolonged period is necessary to perform the test and not because the dye is employed in the execution of the "neutral red test."

The Blood Pressure Curve in Untreated Cases of Hypertension. By DoN C. Sutron, M.D., and (by invitation) Samuel Lang, M.D., Chicago, Ill.

During the past four years a special study has been made of cardiac patients with hypertension, in the Cardiac Clinic at Cook County Hospital. Of this group, sixty-four patients, forty-four of whom were men, have been segregated for study as normal controls. They have received no medical treatment except occasional digitalis, and have been given standard directions, with the exception that diets have been adapted to the individual needs of the patient. These patients have been studied for an average of fourteen weeks, the longest period being seventy-two weeks. Observations have been made on variation of both systolic and diastolic blood pressure in this group, and from this study we have evolved a standard method of control for use in our clinic. Further studies of various methods of treatment will be made. As an illustration of this method of control, it is compared with a series of cases treated with potassium thiocyanate. 
Some Clinical-Pathologic Correlations of Adherent Pericarditis. By HARRY L. Smith, M.D. (by invitation), and Fredrick A. Willius, M.D., Rochester, Minn.

This study comprises 144 cases of adherent pericarditis coming to necropsy at the Mayo Clinic. The pericardial lesions were not minimal but in all instances were well marked examples of the disease.

Age and sex. The great majority of the cases occurred in the fourth, fifth, sixth and seventh decades of life, 108 cases (75 per cent). The youngest patient was two years of age and the oldest patient 85 years of age. The average age was 48.8 years.

A marked dominance in males occurred, 100 cases ( 69.4 per cent), while the female incidence was only 44 cases ( 30.6 per cent).

Pathologic groups. The cases were divided into three groups according to the character of the adherent process. Complete sac obliteration 53 cases ( 36.8 per cent), partial sac obliteration 79 cases ( 54.9 per cent) and parietal adhesions 12 cases ( 8.3 per cent). Calcification of the pericardium occurred in 11 cases (7.6 per cent).

Heart weights. Well marked cardiac hypertrophy was the rule, the average heart weight of the entire group (weights recorded in 106 cases) was 478.1 grams (average normal weight 290 grams).

The greatest heart weight occurred in those cases with partial sac obliteration where the average was 506.5 grams. Where the pericardial sac was completely obliterated, the average heart weight was 472.7 grams and where parietal adhesions occurred the average weight was 251.2 grams. One heart in this latter group weighed 1925 grams inclusive of the pericardium, a chronic tuberculous pericarditis, which was excluded in this average computation owing to its great variation from the other group components.

Associated pathologic cardiac changes. Associated pathologic changes in the heart occurred in 77 cases (53.5 per cent). Their occurrence, in order of frequency, was as follows: Coronary sclerosis, 31 cases (21.5 per cent); rheumatic heart disease with mitral stenosis, 25 cases (17.4 per cent); hypertensive heart disease, 11 cases (7.6 per cent); rheumatic heart disease with aortic insufficiency, 6 cases ( 4.2 per cent) and aortic syphilis, 4 cases (2.8 per cent). The heart weights of these groups are compared.

Associated intrathoracic pathologic changes. Twenty-five cases (17.3 per cent) had associated intrathoracic disease which is discussed.

Mode of death. The heart was solely responsible for death in 57 cases (39.5 per cent), while causes entirely independent of the heart caused death in the remaining 87 cases (60.5 per cent). This data is discussed in relation to the various forms of associated cardiac disease. The average heart weight of the patients dying of heart disease was 625.7 grams, while those dying of other causes had an average heart weight of 411.6 grams. The age factors in relation to death are included in the discussion.

Parenteral Use of Liver Extract in the Treatment of Anemia. By PauL J. Fouts, M.D. (by invitation), and L. G. ZerFas, M.D., Indianapolis, Ind.

Eight patients with anemia, six of whom had primary pernicious anemia, were treated with liver extract administered intravenously. The initial red blood cell levels in five of the cases which showed a reticulocyte response ranged between 1.13 and 3.65 million per cubic millimeter of blood. The maximum reticulocyte response was reached on the fifth day, following the first injection of an amount of liver extract derived from one hundred grams of whole liver. 
One patient with hemolytic jaundice failed to respond satisfactorily to two blood transfusions, to liver extract derived from 600 grams of liver given daily for thirty days, or to $\mathbf{0 . 1 8}$ gram of metallic iron (iron ammonium citrate) administered daily for fourteen days. When liver extract derived from 100 grams of liver was given intravenously, a typical response of the reticulocytes occurred, followed by a satisfactory return of the red blood cells to a normal level.

The comparative potency of liver extract given by injection is discussed, with certain indications for the use of this method.

The Effect of Diacetone Alcohol on the Liver of Rats. By Haddow M. KeIth, M.B., Rochester, Minn.

Redistilled diacetone alcohol was administered by stomach tube to white rats, in doses of $2 \mathrm{cc}$. per kilogram body weight. Pairs of rats were sacrificed at intervals of from one hour to 60 days. Sections of liver were fixed for 24 hours in a Zenker-formol solution, stained with hematoxylin and eosin and with eosin azure II. Four animals were sacrificed as controls.

A distinct drop in the blood hemoglobin and in the red cell count was noted in 24 hours. This persisted for a period of four to five days. On the sixth day both were again approximately normal.

Examination of sections of the liver showed an early increase in lymphocytes and histocytes, followed by vacuolization and granulation of the hepatic cell cytoplasm commencing in the portal zone. This latter was accompanied by a decrease in the histocytic reaction. The maximum hepatic cell injury occurred within 24 hours when the entire lobule was involved. Recovery was first evident at 48 hours, mainly about the central veins, and within 96 hours was relatively complete. At seven and fourteen days, however, there was still slight cytoplasmic granulation in the portal areas, with definite histocytic reaction still present. At twenty-one days, the Kupffer cells were exceedingly abundant, and nests of histocytes were very numerous. The hepatic cells were normal. In thirty-five days recovery was complete.

On the Value of the Electrocardiograph as an Aid in the Diagnosis of Adhesive Mediastino-Pericarditis. By Julius Jensen, M.D., and (by invitation) Milton Smith, M.D., and E. D. Cartwright, M.D., St. Louis, Mo.

In an analysis of fifty normal individuals absence of shift with position in the complexes of the electrocardiograph occurred so frequently as to suggest that the Dieuade sign is of little value in the diagnosis of adhesive mediastinopericarditis.

Comparison of Serum Inorganic Sulphates and Blood Urea Clearance in Renal Insufficiency. By E. G. Wa kefield, M.D., M. H. Power, Ph.D. (by invitation), and Norman M. KeIth, M.D., Rochester, Minn.

The determination of serum inorganic sulphates as a renal functional test has been compared with urea clearance. There is a fairly close correlation between the two as a measure of early renal insufficiency. Previous studies have indicated that serum inorganic sulphate is usually increased (1) before there is retention of either urea or creatinine; (2) before there is lowered excretion of phenolsulphonephthalein, and (3) in about half the subjects studied, sulphates were elevated in the serum before the kidney had lost its ability to concentrate urine to a specific gravity of 1.025 or more in the concentration test; also, the kidney might be unable to concentrate urine to 1.025 and the sulphates would be within the normal range of concentration. These tests do not replace, but supplement other tests of renal functional activity. 
The Determination of Inorganic and Conjugated Sulphates in Urine and Blood Serum. By M. H. Power, Pr.D. (by invitation), and E. G. WAKefield, M.D., Rochester, Minn.

The method involves the preparation of trichloracetic acid filtrates of urine or of serum. From these the sulphate is precipitated by the addition of a solution of benzidine in acetone. The resulting benzidine sulphate is centrifuged down, washed, and quantitatively oxidized by means of concentrated sulphuric acid and a known amount of potassium dichromate. Iodimetric titration of the residual dichromate then supplies the data from which the SO, content of the sample may be calculated. The method has been found satisfactory for amounts ranging from 0.03 to $4.0 \mathrm{mgm}$. of $\mathrm{SO}_{4}$.

Conjugated sulphates are determined in an exactly similar manner after preliminary hydrolysis. The technique developed has been found applicable to the study of the ethereal sulphates in urine as judged by comparison with the results of the Folin gravimetric method. Similar studies of blood serum indicate the ethereal sulphates are present, but not in the amounts which have previously been reported. 